depletion of $\mathrm{CD}_{11 \mathrm{c}^{+}}$cells led to a significant reduction of synovial inflammation and a complete depletion of osteoclasts. Conclusions These data show that in addition to initiating an adaptive immune response, $\mathrm{CD} 11 \mathrm{c}^{+}$dendritic cells, are also involved in innate effector mechanisms of inflammatory arthritis. Especially $\mathrm{CD} 11 \mathrm{~b}^{+} \mathrm{CD} 11 \mathrm{c}^{+}$and monocyte derived inflammatory seem to play a role in inflammatory arthritis, suggesting that they could be an important therapeutic target for patients suffering from inflammatory arthritis.

Disclosure of interest None declared

\section{P057 EFFECTS OF ANTI-TNF THERAPY ON VASCULAR BIOMARKER LEVELS IN RHEUMATOID ARTHRITIS}

'A Hamar*, 'E Végh, 'Á Horváth, 'S Szántó, '⿳G Szucs, 'A Pusztai, 'A Domján, ${ }^{2} \mathrm{~K}$ Hodosi, ${ }^{2} \mathrm{G}$ Kerekes, ${ }^{3} \mathrm{R}$ Gesztelyi, ${ }^{4}$ J Zsuga, ${ }^{5} \mathrm{Z}$ Prohászka, ${ }^{1} \mathrm{Z}$ Szekanecz. ${ }^{1}$ Department of Medicine, Division of Rheumatology; ${ }^{2}$ Department of Medicine; ${ }^{3}$ Department of Pharmacology and Pharmacotherapy; ${ }^{4}$ Department of Health Systems Management and Quality Management for Health Care, University of Debrecen, Debrecen; ${ }^{5}$ Third Department of Internal Medicine, Semmelweis University, Budapest, Hungary

\subsection{6/annrheumdis-2018-EWRR2018.76}

Introduction Prevoius studies have shown an increased risk of cardiovascular disease in rheumatoid arthritis (RA), due to RAassociated inflammation. Different vascular biomarkers, such as anti-hsp65 antibodies, asymmetric dimethylarginine (ADMA) and B-type natriuretic peptide (BNP) have been associated with atherosclerosis and RA. Anti-hsp65 antibodies were found in RA patients and these antibodies linked also to inflammation and atherosclerosis. ADMA is an endogenous competitive inhibitor of NOS and consequential can lead to increased nitrosative and oxidative stress. ADMA has been implicated with atherosclerosis, and also with rheumatoid arthritis. BNP also associated to cardiovascular diseases.

Objectives The aim of this study was to assess the effects of anti-TNF therapy on different vascular biomarkers, such as anti-hsp65, ADMA and BNP in patients with RA and their correlation with different laboratory and clinical markers.

Methods Altogether 36 RA patients were recruited and treated with either etanercept (ETN) or certolizumab pegol (CZP) in this 12 months follow-up study. Assessments were performed at baseline, at month 6 and 12. Amounts of IgG antibodies reacting with recombinant $\mathrm{M}$. bovis hsp65 (Lionex, Braunschweig, Germany) were measured by ELISA. ADMA was assesed by HPLC with fluorescent detection. BNP fragments were assessed by commercially avaiable ELISA kit (Biomedica, Vienna). In addition, disease activity (DAS28), age, disease duration, CRP, IgM rheumatoid factor (RF), anti-CCP (aCCP) and plasma lipid levels were also measured. Arterial flowmediated vasodilation (FMD), carotid intima-media thickness (cIMT) and arterial pulse-wave velocity (PWV) were assessed by ultrasound.

Results There were no significant changes in the levels of antihsp60, ADMA and BNP due to anti-TNF therapy. However, baseline level of BNP is correlated with the levels of RF $(\mathrm{R}=0.479, \mathrm{p}=0.004)$ and $\mathrm{aCCP}(\mathrm{R}=0.591, \mathrm{p}<0.001)$. Serum BNP levels at baseline and at month 6 were significantly increased in RF positive compared to RF negative patients $(680.60 \pm 381.64$ versus $292.94 \pm 198.27 \mathrm{pmol} / \mathrm{L} ; \mathrm{p}=0.007$ and $668.95 \pm 346.51$ versus $312.20 \pm 207.01 \mathrm{pmol} / \mathrm{L} ; \mathrm{p}=0.001)$ and also in aCCP postive compared to aCCP negative patients $(670.61 \pm 323.04$ versus $137.98 \pm 436.41 \mathrm{pmol} / \mathrm{L} ; \mathrm{p}=0.030$ and $652.93 \pm 283.21$ versus $\quad 456.48 \pm 423.11 \mathrm{pmol} / \mathrm{L} ; \quad \mathrm{p}=0.021)$.
Furthermore we found the following correlations between baseline values: anti-hsp60 level correlated with ADMA $(\mathrm{R}=0.900, \mathrm{p}=0.037)$, triglyceride $(\mathrm{TG}) \quad(\mathrm{R}=0.462, \mathrm{p}=0.040)$ and PWV $(\mathrm{R}=0.564, \mathrm{p}=0.040)$. Baseline level of ADMA positively correlated with body mass index $(B M I)$ score $(R=0.720$, $\mathrm{p}=0.040$ ) and also with HDL levels (high density lipoprotein) of patients $(\mathrm{R}=0.473, \mathrm{p}=0.047)$.

Conclusions BNP levels were significantly higher in $\mathrm{RF}+$ compared to RF- patients, which imply that BNP may associate with RF positivity. Specific biomarkers, such as ADMA, anti-hsp60 and BNP may play important role cardiovascular disease in RA.

Acknowledgements The work is supported by the GINOP2.3.2-15-2016-00015/I-KOM TEAMING project.

Disclosure of interest None declared

\section{\begin{tabular}{|l|l}
\hline P058 S100 PROTEINS EFFECTIVELY DISCRIMINATE SYSTEMIC \\
\hline
\end{tabular} LUPUS ERYTHEMATOSUS PATIENTS FROM HEALTHY CONTROLS, BUT ARE NOT ASSOCIATED WITH MEASURES OF DISEASE ACTIVITY}

1,2B Šumová* ${ }^{1,2}$ J Závada, ${ }^{2}$ LA Cerezo, ${ }^{3} \mathrm{M}$ Uher, ${ }^{2} \mathrm{H}$ Hulejová, ${ }^{4} \mathrm{M}$ Grigorian, ${ }^{1,2} \mathrm{~K}$ Pavelka, 1,2J Vencovský, 1,2 L Šenolt. 'Department of Rheumatology, 1st Faculty of Medicine, Charles University, Prague, Czech Republic; ${ }^{2}$ Institute of Rheumatology, Prague, Czech Republic, Prague; ${ }^{3}$ Institute of Biostatistics and Analyses, Masaryk University, Brno, Czech Republic; ${ }^{4}$ Department of Neuroscience, University of Copenhagen, Copenhagen, Denmark

\subsection{6/annrheumdis-2018-EWRR2018.77}

Introduction The S100 proteins are important regulators of diverse calcium-dependent cellular processes including growth regulation, migration and apoptosis. Dysregulated expression of multiple members of S100 family is a common feature of cancer and several autoimmune diseases.

Objectives The aim of this study was to examine whether circulating levels of S100A4, S100A8/9 and S100A12 proteins could be useful as diagnostic or activity specific markers in systemic lupus erythematosus (SLE).

Methods S100 plasma levels were measured by ELISA in a cohort study of 44 patients with SLE, 8 patients with incomplete SLE (iSLE) and 43 healthy controls (HCs). Disease activity was assessed using SLEDAI-2 K. We examined cross sectional associations between concentrations of S100 proteins and SLE status, SLEDAI-2 K scores, and levels of conventional biomarkers.

Results Plasma levels of all analysed S100 proteins (S100A4, S100A8/9 and S100A12) were significantly higher in SLE patients compared to HCs $(\mathrm{p}<0.001, \mathrm{p}<0.01, \mathrm{p}<0.001$, respectively). In iSLE patients, the levels of S100A4 and S100A12 but not S100A8/9 were significantly higher compared to HCs $(\mathrm{p}<0.001, \mathrm{p}<0.05, \mathrm{p}=\mathrm{ns}$, respectively). ROC curve analysis was performed to establish the optimal threshold to discriminate SLE patients from HCs based on S100 levels. At the optimal cutoff point of $238 \mathrm{ng} / \mathrm{ml}$, the area under curve (AUC) for S100A4 was 0-990 $(95 \%$ CI: 0.977 to $1.000, \mathrm{p}<0.001)$ with a sensitivity of $96 \%$ and specify of 93.0\%. The remaining two proteins also showed significant, but not as strong discriminative value [S100A8/9: AUC 0.684 (95\% CI: 0.572 to $0.795, \mathrm{p}<0.05)$; S100A12: AUC 0.809 (95\% CI: 0.719 to $0.898, \mathrm{p}<0.001]$. We found that only S100A12 levels were significantly associated with the SLEDAI$2 \mathrm{~K}$ score $(\mathrm{r}=0.318, \mathrm{p}=0.035)$. Both $\mathrm{S} 100 \mathrm{~A} 8 / 9$ and $\mathrm{S} 100 \mathrm{~A} 12$ levels were significantly higher in SLE patients with arthritis $(\mathrm{p}=0.043, \mathrm{p}=0.015$, respectively) and with haematological 
features $(p=0.002$ for both). There were no other significant associations between S100 levels and other conventional markers such as C3, C4 and the anti-dsDNA antibodies.

Conclusions We demonstrate increased S100 proteins expression in SLE patients. Although they were not strongly associated with disease activity, S100 proteins and namely S100A4 could be proposed as a diagnostic biomarker for SLE.

Acknowledgements MHCR for conceptual development of research organisation 00023728 and SVV 260373.

Disclosure of interest None declared

\section{P059 EX VIVO COMPARISON OF BARICITINIB, UPADACITINIB, FILGOTINIB AND TOFACITINIB FOR CYTOKINE SIGNALLING IN HUMAN LEUKOCYTE SUBPOPULATIONS}

${ }^{1}$ IB Mclnnes, ${ }^{2} \mathrm{R}$ Higgs, ${ }^{2} \mathrm{~J}$ Lee, ${ }^{2} \mathrm{WL}$ Macias, ${ }^{2} \mathrm{~S} \mathrm{Na},{ }^{2} \mathrm{RA}$ Ortmann, ${ }^{2} \mathrm{G}$ Rocha, ${ }^{3} \mathrm{~T}$ Wehrman, ${ }^{2} \mathrm{X}$ Zhang, ${ }^{2} \mathrm{SH}$ Zuckerman, ${ }^{4} \mathrm{PC}$ Taylor, ${ }^{5} \mathrm{C}$ Perrier* ${ }^{1}$ 'Division of Immunology, Infection and Inflammation, University of Glasgow, Glasgow, UK; ${ }^{2}$ Eli Lilly and Company, Indianapolis; ${ }^{3}$ Primity Bio, Fremont, USA; ${ }^{4}$ Medical Sciences Division, University of Oxford, Oxford, UK; ${ }^{5}$ Presenting on behalf of Eli Lilly and Company, Indianapolis, USA

\subsection{6/annrheumdis-2018-EWRR2018.78}

Introduction Baricitinib (BARI), an oral selective Janus kinase (JAK) $1 / 2$ inhibitor, approved in the $\mathrm{EU}$ for moderate to severe active RA.

Objectives To compare in vitro cellular pharmacology of BARI to upadacitinib (ABT), filgotinib (FILGO), and tofacitinib (TOFA), three JAK inhibitors (JAKis) currently approved or in clinical development.

Methods Peripheral blood mononuclear cells from healthy donors $(n=6-12)$ were incubated with different JAKis. After cytokine stimulation, phosphorylated signal transducer and activator of transcription (pSTAT) levels were measured and $\mathrm{IC}_{50}$ calculated in phenotypically gated leukocyte subpopulations. Therapeutic dose relevance of in vitro analysis was assessed using calculated mean concentration-time (CT) profiles over 24 hour JAKi-treated subjects (BARI $4 \mathrm{mg}$ QD; ABT 15 and $30 \mathrm{mg}$ QD; FILGO 100 and $200 \mathrm{mg}$ QD; TOFA 5 and $10 \mathrm{mg}$ BID). Time above $\mathrm{IC}_{50}(\mathrm{~T}>\mathrm{IC} ; \mathrm{h} /$ day) and average daily $\%$ inhibition of pSTAT formation (\%SI) were calculated for each JAKi, cytokine, and cell type.

Results Tested cytokines did not signal in all cell types. When signalling was detected, $\mathrm{IC}_{50}, \% \mathrm{SI}$, and $\mathrm{T}>\mathrm{IC}$ for a particular JAKi exhibited similar dose dependent inhibition across cell types. For JAK1/3 dependent signalling across 4 cytokines (IL$2,4,15,21), \mathrm{IC}_{50}$ for $\mathrm{ABT}$ and TOFA were more potent than BARI; FILGO was the least potent. Overlaid on CT profiles, this indicated generally higher\%SI and longer $\mathrm{T}>\mathrm{IC}$ for ABT and TOFA compared to BARI and FILGO. For IL-6 (JAK1/2),\%SI and T>IC was TOFA $>$ BARI/ABT $>$ FILGO and for IL-10 (JAK1/TYK2),\%SI was TOFA $>$ BARI/ABT $>$ FILGO. IFN- $\gamma(\mathrm{JAK} 1 / 2)$ was modulated only by BARI, ABT, and TOFA. IFN- $\alpha$ (JAK1/TYK2) signalling was most potently inhibited by BARI and ABT. FILGO did not appear to modulate GM-CSF signalling (JAK2/2), while $\%$ SI and $\mathrm{T}>\mathrm{IC}$ were similar between BARI and ABT.

Conclusions JAKis modulate distinct cytokine pathways to differing degrees and durations over 24 hour. BARI and FILGO inhibited JAK1/3 signalling less than ABT and TOFA. No JAKis agent potently or continuously inhibited an individual cytokine signalling pathway throughout dosing interval, implying the varying efficacy and safety profiles of JAKis across disease states.

Acknowledgements
Study support Eli Lilly and Company and Incyte Corporation. Encore of ACR/ARHP-2017 Annual Scientific Meeting, Nov 4-8, 2016; San Diego, CA, USA.

Disclosure of interest None declared

\section{P060 IL-38 IN ARTHRITIS MATURATION AND DEGRADATION OF THIS NEW IL-1 FAMILY ANTI-INFLAMMATORY CYTOKINE}

M Harel, T Garraud, B Le Goff, F Blanchard*. 'INSERM UMR1238 Rheumatology unit, Nantes University Hospital, Nantes, France

\subsection{6/annrheumdis-2018-EWRR2018.79}

Introduction Interleukin 38 (IL-38) is an IL-1 family cytokine with potential anti-inflammatory activity. Recently we showed that over-expression of full length IL-38 reduces inflammation in arthritic mice by $20 \%-30 \%$, reduces macrophage infiltration and the expression of cytokines implicated in the biology of Th17 cells. ${ }^{1}$ However, IL-38 does not have a classical signal peptide, and it is unclear how it is released for example from monocytes/macrophages. It needs post-translational processing (N-terminal truncation) to acquire full biological activity, but the required proteases are unknown.

Objectives The aim is to study the maturation of IL-38 in the inflammatory process.

Methods THP1 macrophagic cells were transduced with a lentivirus encoding full length IL-38 to over-express this cytokine. Secretion and maturation (truncation) of endogenous or overexpressed IL-38 has been studied by western blot. The potential proteases implicated in IL-38 N-terminal truncation have been analysed in silico (Prosper webserver) and by biochemical tests using recombinant proteases and their inhibitors.

Results In the synovial fluid of RA patients, we observed 2 truncated forms of IL-38. To determine if apoptosis is a mechanism responsible for the maturation of IL-38, we tested different stress conditions on THP1 macrophages that overexpress IL-38. In conditioned media, three truncated forms of IL-38 were revealed by Western Blot under conditions of apoptosis, exacerbated oxidative stress, or necrosis. However, none of these conditioned media appears to have a high antiinflammatory effect on macrophages. Our results rather suggest that cell death by apoptosis or necrosis results in IL-38 degradation and loss of biological effect. In silico, several potential cleavage sites were found for calpain, MMP-2, MMP-9 and cathepsin G. Incubation of recombinant full length IL-38 with MMP-2 or cathepsin G leads to significant degradation of this cytokine. On apoptotic THP1 cells overexpressing IL-38, an inhibitor of MMPs and TACE (TAPI) also reduces the release and degradation of IL-38.

Conclusions The degradation and loss of the anti-inflammatory effect of IL-38 appears to occur during cell death in the extracellular space, and could involve MMPs. The processes involved in the maturation of IL-38 need to be further explored and could open new therapeutic opportunities for the treatment of arthritis.

\section{REFERENCE}

1. Boutet MA, et al. IL-38 overexpression induces anti-inflammatory effects in mice arthritis models and in human macrophages in vitro. Ann Rheum Dis 2017;76:1304-1312.

Disclosure of interest M. Harel: None declared, T. Garraud: None declared, B. Le Goff: None declared, F. Blanchard 